\title{
Least energy nodal solution of a singular perturbed problem with jumping nonlinearity
}

\author{
Edward N. DANCER, SAnjiban SANTra And Juncheng Wei
}

\begin{abstract}
In this paper we study the asymptotic behavior of the least energy nodal solution of a problem with a jumping nonlinearity.
\end{abstract}

Mathematics Subject Classification (2010):35J10 (primary); 35J65 (secondary).

\section{Introduction}

There has been a considerable interest to understand the asymptotic behavior of positive solutions of the elliptic problem

$$
\begin{cases}\varepsilon^{2} \Delta u-u+f(u)=0 & \text { in } \Omega \\ u=0 & \text { on } \partial \Omega\end{cases}
$$

where $\varepsilon>0$ is a parameter, $f$ is a superlinear function, $\Omega$ is a smooth bounded domain in $\mathbb{R}^{N}$. Let $F(u)=\int_{0}^{u} f(t) d t$. In this paper, we consider the problem

$$
\begin{cases}\varepsilon^{2} \Delta u-\lambda_{1} u^{+}+\lambda_{2} u^{-}+f(u)=0 & \text { in } \Omega \\ u^{ \pm} \neq 0 & \text { in } \Omega \\ u=0 & \text { on } \partial \Omega\end{cases}
$$

where $\lambda_{1}>0, \lambda_{2}>0$ with $\lambda_{1} \neq \lambda_{2}$, and $u^{ \pm}=\max \{ \pm u, 0\}$. Let $f: \mathbb{R} \rightarrow \mathbb{R}$ be a continuously differentiable function satisfying:

(f1) $f(t)=o(t)$ as $t \rightarrow 0$;

(f2) $f(t)=O\left(|t|^{p}\right)$ as $t \rightarrow+\infty$ for some $p \in\left(1, \frac{N+2}{N-2}\right)$ if $N \geq 3$ and $p>1$ if $N=1,2$;

The first and the second author were supported by ARC and the third author was supported by an Earmarked grant from RGC of Hong Kong.

Received November 2, 2009; accepted December 10, 2009. 
(f3) there exists a constant $\theta>2$ such that $\theta F(t) \leq t f(t)$ where

$$
F(t)=\int_{0}^{t} f(s) d s
$$

(f4) $|t| f^{\prime}(t)>f(t)(\operatorname{sgn} t)$ for all $t \neq 0$.

Condition (f4) implies that $\frac{1}{2} f(t) t-F(t)$ is strictly increasing in $(0,+\infty)$. Problem (1.1) arises in various applications, such as chemotaxis, population genetic, chemical reactor theory. Problem (1.2) arises in the study of population dynamics with jumping nonlinearity [9]. It can also be considered as the limiting problem of the following elliptic system

$$
\begin{cases}\varepsilon^{2} \Delta u-\lambda_{1} u+\mu_{1} u^{3}+\beta u v^{2}=0 & \text { in } \Omega \\ \varepsilon^{2} \Delta v-\lambda_{2} v+\mu_{2} v^{3}+\beta v u^{2}=0 & \text { in } \Omega \\ u, v>0 & \text { in } \Omega \\ u=v=0 & \text { on } \partial \Omega\end{cases}
$$

The system (1.3) arises in the Bose-Einstein condenstates and nonlinear optics. An important phenomena of (1.3) is the so-called phase separation. As $\beta \rightarrow-\infty$, the components $u, v$ separates and the difference function $u-v$ approaches a solution of (1.2) with $f(u)=\mu_{1} u_{+}^{3}-\mu_{2} u_{-}^{3}$. This has been proved for the least energy solution of $(1.3)$ in $[5,7]$ and for radial solutions on two dimensional balls in [20]. We refer to $[1,2,4,5,8,10,14,19,20]$ and the references therein.

Existence and concentration of positive solution of this type of problems were extensively studied by Ni-Takagi [16,17], Ni-Wei [18], del Pino- Felmer [11].

Define

$$
I_{\lambda_{1}}(W)=\frac{1}{2} \int_{\mathbb{R}^{N}}|\nabla W|^{2}+\frac{\lambda_{1}}{2} \int_{\mathbb{R}^{N}} W^{2}-\int_{\mathbb{R}^{N}} F(W)
$$

and

$$
I_{\lambda_{2}}(W)=\frac{1}{2} \int_{\mathbb{R}^{N}}|\nabla W|^{2}+\frac{\lambda_{2}}{2} \int_{\mathbb{R}^{N}} W^{2}-\int_{\mathbb{R}^{N}} F(W) .
$$

Let $W_{\lambda_{1}}$ be a least energy positive solution of

$$
\begin{cases}-\Delta u+\lambda_{1} u=f(u) & \text { in } \mathbb{R}^{N} \\ u>0 & \text { in } \mathbb{R}^{N} \\ u \in H^{1}\left(\mathbb{R}^{N}\right) & \end{cases}
$$

and $W_{\lambda_{2}}$ be a least positive solution of

$$
\begin{cases}-\Delta u+\lambda_{2} u=f(u) & \text { in } \mathbb{R}^{N} \\ u>0 & \text { in } \mathbb{R}^{N} \\ u \in H^{1}\left(\mathbb{R}^{N}\right) . & \end{cases}
$$


By Gidas, Ni and Nirenberg [13], it is well known that $W_{\lambda_{i}}$ is radially decreasing and decays as

$$
W_{\lambda_{i}}(|x|) \sim e^{-\sqrt{\lambda_{i}}|x|}|x|^{\frac{1-N}{2}} \text { as }|x| \rightarrow+\infty
$$

for $i=1,2$. Throughout the course of the paper we will call $W_{\lambda_{i}}$ an entire solution or a ground state.

In this paper, we prove the existence of a least energy nodal solution and show that for $\varepsilon$ sufficiently small, the solution has a exactly one positive spike and one negative spike and the spikes concentrate at two distinct points of $\Omega$, in other words they repel each other. We define a function $\varphi: \Omega \times \Omega \rightarrow \mathbb{R}$ by

$$
\left.\varphi(x, y)=\min \left\{\sqrt{\lambda_{1}} d(x, \partial \Omega), \sqrt{\lambda_{2}} d(y, \partial \Omega)\right), \frac{1}{2} \frac{\sqrt{\lambda_{1}} \sqrt{\lambda_{2}}}{\sqrt{\lambda_{1}}+\sqrt{\lambda_{2}}}|x-y|\right\} .
$$

Theorem 1.1. There exists $\varepsilon_{0}>0$ such that for every $0<\varepsilon<\varepsilon_{0}$, the least energy nodal solution $u_{\varepsilon} \in H_{0}^{1}(\Omega)$ of (1.2) having exactly one positive local maximum (hence a global maximum) point $P_{\varepsilon}^{1}$ and one negative local minimum (hence a global minimum) point $P_{\varepsilon}^{2}$ and

$$
\lim _{\varepsilon \rightarrow 0} \varphi\left(P_{\varepsilon}^{1}, P_{\varepsilon}^{2}\right)=\max _{(x, y) \in \bar{\Omega} \times \bar{\Omega}} \varphi(x, y),
$$

with $u_{\varepsilon}\left(P_{\varepsilon}^{i}\right) \rightarrow(-1)^{i-1} W_{\lambda_{i}}(0)$ and $u_{\varepsilon} \rightarrow 0$ in $\mathcal{C}_{\text {loc }}^{1}\left(\Omega \backslash\left\{P_{\varepsilon}^{1}, P_{\varepsilon}^{2}\right\}\right)$.

Note that for sufficiently small $\varepsilon>0$, the least energy positive solution to the problem (1.1) has a unique maxima $P_{\varepsilon} ; u_{\varepsilon}$ decays exponentially away from $P_{\varepsilon}$ and $d\left(P_{\varepsilon}, \partial \Omega\right) \rightarrow \max _{P \in \Omega} d(P, \partial \Omega)$ as $\varepsilon \rightarrow 0$, which implies that the solution concentrates at an interior point furthest from the boundary of $\Omega$. This was studied by $\mathrm{Ni}-$ Wei [15]. For the least energy nodal solution, the problem was studied by Noussair-Wei [18] when $\lambda_{1}=\lambda_{2}=1$ and $f(u)=u^{p}$. They obtain the same results as in Theorem 1.1. In addition, they prove that $u_{\varepsilon}(x)=W\left(\frac{x-P_{\varepsilon}^{1}}{\varepsilon}\right)-W\left(\frac{x-P_{\varepsilon}^{2}}{\varepsilon}\right)+v_{\varepsilon}$, where $\left\|v_{\varepsilon}\right\|_{L^{\infty}(\Omega)} \rightarrow 0$ as $\varepsilon \rightarrow 0$ and $W$ is the unique solution of the limiting problem. The study of asymptotic behavior involves the uniqueness and non-degeneracy of solution of the limiting problem. Then using the expansion, an asymptotic expansion of the energy is obtained. This approach does not work here since $u_{+}$and $u_{-}$are not differentiable. Neither we have uniqueness nor nondegeneracy of the ground state. There is another approach by del Pino and Felmer [11] where they used variational characterizations of positive solutions and symmetrization technique. However their approach works well for positive solutions but does not work for sign-changing solutions. We shall modify the approach of del Pino and Felmer. The problem here is more complicated since the solution is sign-changing and we have to estimate the interaction of the positive and negative components. 


\section{Preliminaries}

Without loss of generality, we consider $0<\lambda_{1}<\lambda_{2}$. The associated functional to the problem (1.2) is

$$
E_{\varepsilon}(u)=\int_{\Omega}\left(\frac{\varepsilon^{2}}{2}|\nabla u|^{2}+\frac{\lambda_{1}}{2}\left(u^{+}\right)^{2}+\frac{\lambda_{2}}{2}\left(u^{-}\right)^{2}-F(u)\right) d x .
$$

Note that from $\left(f_{2}\right), E_{\varepsilon} \in \mathcal{C}^{1}\left(H_{0}^{1}(\Omega), \mathbb{R}\right)$. Moreover, if $u_{\varepsilon} \in H_{0}^{1}(\Omega)$ is a critical point of $E_{\varepsilon}$, then $u_{\varepsilon} \in \mathcal{C}^{2}(\Omega) \cap \mathcal{C}(\bar{\Omega})$ and hence $u_{\varepsilon}$ is a classical solution of (1.2). Note that $E_{\varepsilon}(u)=E_{\varepsilon, \lambda_{1}}(u)+E_{\varepsilon, \lambda_{2}}(u)$ where

$$
\begin{aligned}
& E_{\varepsilon, \lambda_{1}}(u)=\int_{\Omega}\left(\frac{\varepsilon^{2}}{2}\left|\nabla u^{+}\right|^{2}+\frac{\lambda_{1}}{2}\left(u^{+}\right)^{2}-F\left(u^{+}\right)\right) d x, \\
& E_{\varepsilon, \lambda_{2}}(u)=\int_{\Omega}\left(\frac{\varepsilon^{2}}{2}\left|\nabla u^{-}\right|^{2}+\frac{\lambda_{2}}{2}\left(u^{-}\right)^{2}-F\left(u^{-}\right)\right) d x .
\end{aligned}
$$

Define the Nehari set as

$$
\begin{array}{r}
\mathcal{N}_{\varepsilon}=\left\{u \in H_{0}^{1}(\Omega): u^{ \pm} \not \equiv 0, \varepsilon^{2} \int_{\Omega}\left|\nabla u^{+}\right|^{2}+\lambda_{1} \int_{\Omega}\left(u^{+}\right)^{2}=\int_{\Omega} f\left(u^{+}\right) u^{+} ;\right. \\
\left.\varepsilon^{2} \int_{\Omega}\left|\nabla u^{-}\right|^{2}+\lambda_{2} \int_{\Omega}\left(u^{-}\right)^{2}=\int_{\Omega} f\left(u^{-}\right) u^{-}\right\} .
\end{array}
$$

Define the positive and negative Nehari set as

$$
\mathcal{N}_{\varepsilon}^{+}=\left\{u \in H_{0}^{1}(\Omega):\left\langle E_{\varepsilon, \lambda_{1}}^{\prime}(u), u\right\rangle=0 ; u \not \equiv 0 \text { and } u \geq 0\right\}
$$

and

$$
\mathcal{N}_{\varepsilon}^{-}=\left\{u \in H_{0}^{1}(\Omega):\left\langle E_{\varepsilon, \lambda_{2}}^{\prime}(u), u\right\rangle=0 ; u \not \equiv 0 \text { and }-u \geq 0\right\}
$$

respectively. Note that any $u$ belonging to $\mathcal{N}_{\varepsilon}$ is sign-changing. Moreover, all the sign-changing solutions of (1.2) are contained in $\mathcal{N}_{\varepsilon}$. Also note that $\mathcal{N}_{\varepsilon}^{+} \cap \mathcal{N}_{\varepsilon}^{-}=\emptyset$. Let

$$
c_{\varepsilon}=\inf _{u \in \mathcal{N}_{\varepsilon}} E_{\varepsilon}(u)
$$

Remark 2.1. The set $\mathcal{N}_{\varepsilon}$ is not a manifold in $H_{0}^{1}(\Omega)$ due to the lack of differentiability of the map $u \mapsto u^{ \pm}$. In fact, $\mathcal{N}_{\varepsilon} \cap H^{2}(\Omega)$ is a $\mathcal{C}^{1}$ manifold of codimension 2 in $H^{2}(\Omega)$, see [1]. Hence it is not clear whether a minimizer of $E_{\varepsilon}$ on $\mathcal{N}_{\varepsilon}$ is indeed a solution of (1.2).

Remark 2.2. Define $h^{ \pm}(t)=E_{\varepsilon}\left(t u_{\varepsilon}^{ \pm}\right)$. Note that $h^{ \pm}$is strictly increasing for $t \in$ $(0,1)$ and strictly decreasing in $t \in(1,+\infty)$. This implies that $\max _{0<t<+\infty} h^{ \pm}(t)$ exists and occurs at $t=1$. 
We will show that there exists $u_{\varepsilon} \in \mathcal{N}_{\varepsilon}$ such that $c_{\varepsilon}=E_{\varepsilon}\left(u_{\varepsilon}\right)$, and that $u_{\varepsilon}$ is a least energy sign-changing solution. We state some elementary lemmas,

Lemma 2.3. For all $\varepsilon>0, \mathcal{N}_{\varepsilon}^{+}$and $\mathcal{N}_{\varepsilon}^{-}$are closed subsets of $H_{0}^{1}(\Omega)$.

$$
0<c_{\varepsilon}^{+}=\inf _{u \in \mathcal{N}_{\varepsilon}^{+}} E_{\varepsilon, \lambda_{1}}(u)=\inf _{u \in H_{0}^{1}(\Omega), u \neq 0} \max _{t \geq 0} E_{\varepsilon, \lambda_{1}}(t u)
$$

and

$$
0<c_{\varepsilon}^{-}=\inf _{u \in \mathcal{N}_{\varepsilon}^{-}} E_{\varepsilon, \lambda_{2}}(u)=\inf _{u \in H_{0}^{1}(\Omega), u \neq 0} \max _{t \geq 0} E_{\varepsilon, \lambda_{2}}(t u)
$$

Moreover, $\mathcal{N}_{\varepsilon}^{ \pm}$is a $\mathcal{C}^{1}$ manifold of codimension 1 and every minimizer $u$ of $E_{\varepsilon}$ on $\mathcal{N}_{\varepsilon}^{ \pm}$is positive.

Proof. This follows trivially by using $\left(f_{4}\right)$ and Sobolev embedding theorem. See [15]. $\mathcal{N}_{\varepsilon}^{ \pm}$is a $\mathcal{C}^{1}$ manifold of codimension 1 follows from [3].

Lemma 2.4. There exists some $u_{\varepsilon} \in \mathcal{N}_{\varepsilon}$ such that $c_{\varepsilon}$ is achieved. Moreover, $u_{\varepsilon}$ is $a$ weak solution and hence a classical nodal solution of (1.2).

Proof. Let $\varepsilon>0$ be fixed. We use the argument by Bartsch, Weth and Willem [2]. Since $c_{\varepsilon}=\inf _{u \in \mathcal{N}_{\varepsilon}} E_{\varepsilon}(u)$, there exists a minimizing sequence $u_{\varepsilon, n} \in \mathcal{N}_{\varepsilon}$ such that $E_{\varepsilon}\left(u_{\varepsilon, n}\right) \rightarrow c_{\varepsilon}$ as $n \rightarrow+\infty$. Note that by $(f 3), E_{\varepsilon}$ is coercive on $\mathcal{N}_{\varepsilon}$, as

$$
E_{\varepsilon}\left(u_{\varepsilon, n}\right) \geq\left(\frac{1}{2}-\frac{1}{\theta}\right) \int_{\Omega}\left\{\varepsilon^{2}\left|\nabla u_{\varepsilon, n}\right|^{2}+\lambda_{1}\left(u_{\varepsilon, n}^{+}\right)^{2}+\lambda_{2}\left(u_{\varepsilon, n}^{-}\right)^{2}\right\} .
$$

and hence there exist $b(\varepsilon)>0, d(\varepsilon)>0$ independent of $n$ such that $b(\varepsilon) \leq$ $\left\|u_{\varepsilon, n}^{ \pm}\right\|_{H_{0}^{1}(\Omega)} \leq d(\varepsilon)$. Therefore there exist $u_{\varepsilon}^{ \pm} \in H_{0}^{1}(\Omega)$ such that $u_{\varepsilon, n}^{ \pm} \rightarrow u_{\varepsilon}^{ \pm}$ as $n \rightarrow+\infty$ and by the Rellich Lemma $u_{\varepsilon, n}^{ \pm} \rightarrow u_{\varepsilon}^{ \pm}$in $L^{q}(\Omega)$ for $q \in\left(1, \frac{2 N}{N-2}\right)$. This implies that $u_{\varepsilon}^{ \pm} \geq 0$ and $u_{\varepsilon}^{+} \cdot u_{\varepsilon}^{-}=0$ since $u_{\varepsilon, n}^{+} \cdot u_{\varepsilon, n}^{-}=0$. Thus $u_{\varepsilon}^{ \pm}$are indeed the positive and negative part of $u_{\varepsilon}=u_{\varepsilon}^{+}-u_{\varepsilon}^{-}$. From the fact that (2.2) and (2.3) we have $\left\|u_{\varepsilon, n}^{ \pm}\right\|_{L^{q}(\Omega)}$ has a positive lower bound and this implies $u_{\varepsilon}^{ \pm} \not \equiv 0$. But also we have

$$
\lim _{n \rightarrow \infty} \int_{\Omega} f\left(u_{\varepsilon, n}^{ \pm}\right) u_{\varepsilon, n}^{ \pm}=\int_{\Omega} f\left(u_{\varepsilon}^{ \pm}\right) u_{\varepsilon}^{ \pm}
$$

and

$$
\lim _{n \rightarrow \infty} \int_{\Omega} F\left(u_{\varepsilon, n}^{ \pm}\right)=\int_{\Omega} F\left(u_{\varepsilon}^{ \pm}\right)
$$

From (2.6) using Fatou's lemma we have

$$
\left\|u_{\varepsilon}^{ \pm}\right\|_{H_{0}^{1}(\Omega)}^{2} \leq \int_{\Omega} f\left(u_{\varepsilon}^{ \pm}\right) u_{\varepsilon}^{ \pm}
$$


By a variant Remark 2.2 there exist $s, t \in(0,1]$ such that

$$
\left\|t u_{\varepsilon}^{+}\right\|_{H_{0}^{1}(\Omega)}^{2}=\int_{\Omega} f\left(t u_{\varepsilon}^{+}\right) t u_{\varepsilon}^{+}
$$

and

$$
\left\|s u_{\varepsilon}^{-}\right\|_{H_{0}^{1}(\Omega)}^{2}=\int_{\Omega} f\left(s u_{\varepsilon}^{-}\right) s u_{\varepsilon}^{-} .
$$

This implies $t u_{\varepsilon}^{+}-s u_{\varepsilon}^{-} \in \mathcal{N}_{\varepsilon}$ and hence

$$
\begin{aligned}
E_{\varepsilon}\left(t u_{\varepsilon}^{+}-s u_{\varepsilon}^{-}\right) & =E_{\varepsilon, \lambda_{1}}\left(t u_{\varepsilon}^{+}\right)+E_{\varepsilon, \lambda_{2}}\left(s u_{\varepsilon}^{-}\right) \\
& \leq \lim _{n \rightarrow \infty} E_{\varepsilon, \lambda_{1}}\left(u_{\varepsilon, n}^{+}\right)+\lim _{n \rightarrow \infty} E_{\varepsilon, \lambda_{2}}\left(u_{\varepsilon, n}^{-}\right)=c_{\varepsilon}
\end{aligned}
$$

Note that we have used the fact (f4), (2.6), (2.7) to obtain

$$
E_{\varepsilon, \lambda_{1}}\left(t u_{\varepsilon}^{+}\right) \leq \lim _{n \rightarrow \infty} E_{\varepsilon, \lambda_{1}}\left(u_{\varepsilon}^{+}\right) \text {and } E_{\varepsilon, \lambda_{2}}\left(s u_{\varepsilon}^{-}\right) \leq \lim _{n \rightarrow \infty} E_{\varepsilon, \lambda_{2}}\left(u_{\varepsilon}^{-}\right)
$$

Hence we have $c_{\varepsilon} \leq E_{\varepsilon}\left(t u_{\varepsilon}^{+}-s u_{\varepsilon}^{-}\right) \leq c_{\varepsilon}$ and indeed $t u_{\varepsilon}^{+}-s u_{\varepsilon}^{-}$is a minimizer in $\mathcal{N}_{\varepsilon}$.

By Remark 2.1 we want to show that $v_{\varepsilon}:=t u_{\varepsilon}^{+}-s u_{\varepsilon}^{-}$is a critical point of $E_{\varepsilon}$. If possible, let $E_{\varepsilon}^{\prime}\left(v_{\varepsilon}\right) \neq 0$ and then there exist $\delta>0$ and $\lambda>0$ such that

$$
\left\|E_{\varepsilon}^{\prime}(w)\right\| \geq \lambda \text { whenever }\left\|v_{\varepsilon}-w\right\| \leq \delta
$$

Define a square $S=\left(\frac{1}{2}, \frac{3}{2}\right) \times\left(\frac{1}{2}, \frac{3}{2}\right)$ and for any $(m, n) \in S$

$$
\psi(m, n)=m v_{\varepsilon}^{+}-n v_{\varepsilon}^{-} .
$$

Then from (2.8) we have

$$
\tilde{c}_{\varepsilon}=\max _{\partial S} E_{\varepsilon}(\psi)<c_{\varepsilon}
$$

Indeed our earlier comments, $E_{\varepsilon}(\psi)<c_{\varepsilon}$ on $S$ except at $(1,1)$. Choose $\tau=$ $\min \left\{\frac{c_{\varepsilon}-\tilde{c}_{\varepsilon}}{2}, \frac{\lambda \delta}{8}\right\}$ and $B\left(v_{\varepsilon}, \delta\right)$ be ball centered at $v_{\varepsilon}$. Then by Willem [21, Lemma 2.3, page 38], there exist a deformation $\eta \in \mathcal{C}\left([0,1] \times H_{0}^{1}(\Omega) ; H_{0}^{1}(\Omega)\right)$ such that

(a) $\eta(t, w)=w$ if $t=0$ or if $w \in E_{\varepsilon}^{-1}\left(c_{\varepsilon}-2 \tau, c_{\varepsilon}+2 \tau\right)$,

(b) $\eta\left(1, E_{\varepsilon}^{c_{\varepsilon}+\tau} \cap B\left(v_{\varepsilon}, \delta\right)\right) \subset E_{\varepsilon}^{c_{\varepsilon}-\tau}$,

(c) $E_{\varepsilon}(\eta(1, w)) \leq E_{\varepsilon}(w), \forall w \in H_{0}^{1}(\Omega)$. Moreover, by our remarks and results in [21], we have

$$
\max _{(m, n) \in \bar{S}} E_{\varepsilon}\left(\eta(1, \psi(m, n))<c_{\varepsilon} .\right.
$$


The idea of the proof is to obtain a contradiction. To this end we claim that $\eta(1, \psi(S)) \cap \mathcal{N}_{\varepsilon} \neq \emptyset$. Define $h(m, n)=\eta(1, \psi(m, n))$ and

$$
\begin{aligned}
& \Pi_{1}(m, n)=\left(E_{\varepsilon}^{\prime}\left(m v_{\varepsilon}^{+}\right) v_{\varepsilon}^{+}, E_{\varepsilon}^{\prime}\left(n v_{\varepsilon}^{-}\right) v_{\varepsilon}^{-}\right) \\
& \Pi_{2}(m, n)=\left(\frac{1}{m} E_{\varepsilon}^{\prime}\left(h^{+}(m, n)\right) h^{+}(m, n), \frac{1}{n} E_{\varepsilon}^{\prime}\left(h^{-}(m, n)\right) h^{-}(m, n)\right) .
\end{aligned}
$$

Note that the first component of $\Pi_{1}(m, n)$ is positive if $m<1$ and is negative if $m>1$ with an analogous property for the second component. Hence by the product rule for degree theory we have $\operatorname{deg}\left(\Pi_{1}, S, 0\right)=1$. Moreover, as $\psi=h$ on $\partial S$ (by our choice of $\tau$ and the property (a) of the deformation) we must have $\operatorname{deg}\left(\Pi_{1}, S, 0\right)=\operatorname{deg}\left(\Pi_{2}, S, 0\right)$. Hence there exists a tuple $\left(m_{0}, n_{0}\right) \in S$ such that $\Pi_{2}\left(m_{0}, n_{0}\right)=0$ which implies $h\left(m_{0}, n_{0}\right)=\eta\left(1, \psi\left(m_{0}, n_{0}\right)\right) \in \mathcal{N}_{\varepsilon}$.

Lemma 2.5. Let $\omega_{\varepsilon, \lambda_{1}}$ and $\omega_{\varepsilon, \lambda_{2}}$ be the least energy solutions of

$$
\begin{aligned}
& \begin{cases}-\varepsilon^{2} \Delta u+\lambda_{1} u=f(u) & \text { in } B_{r}(0) \\
u>0 & \text { in } B_{r}(0) \\
u=0 & \text { on } \partial B_{r}(0)\end{cases} \\
& \begin{cases}-\varepsilon^{2} \Delta u+\lambda_{2} u=f(u) & \text { in } B_{r}(0) \\
u>0 & \text { in } B_{r}(0) \\
u=0 & \text { on } \partial B_{r}(0)\end{cases}
\end{aligned}
$$

respectively. Then for sufficiently small $\varepsilon>0$, we have

$$
\begin{aligned}
& E_{\varepsilon, \lambda_{1}}\left(\omega_{\varepsilon, \lambda_{1}}\right)=\varepsilon^{N}\left\{I_{\lambda_{1}}\left(W_{\lambda_{1}}\right)+e^{-\frac{2 \sqrt{\lambda_{1}} r(1+o(1))}{\varepsilon}}\right\} \\
& E_{\varepsilon, \lambda_{2}}\left(\omega_{\varepsilon, \lambda_{2}}\right)=\varepsilon^{N}\left\{I_{\lambda_{2}}\left(W_{\lambda_{2}}\right)+e^{-\frac{2 \sqrt{\lambda_{2}} r(1+o(1))}{\varepsilon}}\right\}
\end{aligned}
$$

where $o(1) \rightarrow 0$ as $\varepsilon \rightarrow 0$.

Proof. For the proof see [11].

Let $\Lambda=\left\{x \in \Omega: \sqrt{\lambda_{1}}\left|x-P_{1}\right|=\sqrt{\lambda_{2}}\left|x-P_{2}\right|\right\}$.

Lemma 2.6. We have for $\varepsilon>0$ sufficiently small

$$
c_{\varepsilon} \leq \varepsilon^{N}\left\{I_{\lambda_{1}}\left(W_{\lambda_{1}}\right)+I_{\lambda_{2}}\left(W_{\lambda_{2}}\right)+e^{-\frac{2 \varphi\left(P_{1}, P_{2}\right)}{\varepsilon}}+o\left(e^{-\frac{2 \varphi\left(P_{1}, P_{2}\right)}{\varepsilon}}\right)\right\} .
$$


Proof. Let $v_{\varepsilon}$ be a positive solution of

$$
\begin{cases}-\varepsilon^{2} \Delta u+\lambda_{1} u=f(u) & \text { in } B_{r_{1}}\left(P_{1}\right) \\ u>0 & \text { in } B_{r_{1}}\left(P_{1}\right) \\ u=0 & \text { on } B_{r_{1}}\left(P_{1}\right)\end{cases}
$$

where $r_{1}=\min \left\{d\left(P_{1}, \partial \Omega\right), d\left(P_{1}, \Lambda\right)\right\}$. Let $w_{\varepsilon}$ be a positive solution of

$$
\begin{cases}-\varepsilon^{2} \Delta u+\lambda_{2} u=f(u) & \text { in } B_{r_{2}}\left(P_{2}\right) \\ u>0 & \text { in } B_{r_{2}}\left(P_{2}\right) \\ u=0 & \text { on } B_{r_{2}}\left(P_{2}\right)\end{cases}
$$

where $r_{2}=\min \left\{d\left(P_{2}, \partial \Omega\right), d\left(P_{2}, \Lambda\right)\right\}$. Note that supp $v_{\varepsilon} \cap \operatorname{supp} w_{\varepsilon}=\emptyset$ and $v_{\varepsilon} \in$ $\mathcal{N}_{\varepsilon}^{+}$and $w_{\varepsilon} \in \mathcal{N}_{\varepsilon}^{-}$. Then we have $v_{\varepsilon}-w_{\varepsilon} \in \mathcal{N}_{\varepsilon}$ and hence we have from (2.15) and (2.16),

$$
\begin{aligned}
c_{\varepsilon} & \leq E_{\varepsilon}\left(v_{\varepsilon}-w_{\varepsilon}\right) \\
& \leq E_{\varepsilon, \lambda_{1}}\left(v_{\varepsilon}\right)+E_{\varepsilon, \lambda_{2}}\left(w_{\varepsilon}\right) \\
& \leq \varepsilon^{N}\left\{I_{\lambda_{1}}\left(W_{\lambda_{1}}\right)+e^{-\frac{2 r_{1}}{\varepsilon}}+I_{\lambda_{2}}\left(W_{\lambda_{2}}\right)+e^{-\frac{2 r_{2}}{\varepsilon}}+o\left(e^{-\frac{2 r_{1}}{\varepsilon}}\right)+o\left(e^{-\frac{2 r_{2}}{\varepsilon}}\right)\right\} .
\end{aligned}
$$

Hence we have,

$$
\begin{aligned}
c_{\varepsilon} & \leq \varepsilon^{N}\left\{I_{\lambda_{1}}\left(W_{\lambda_{1}}\right)+e^{-\frac{2 \min \left\{r_{1}, r_{2}\right\}}{\varepsilon}}+I_{\lambda_{2}}\left(W_{\lambda_{2}}\right)+o\left(e^{-\frac{2 \min \left\{r_{1}, r_{2}\right\}}{\varepsilon}}\right)\right\} \\
& \leq \varepsilon^{N}\left\{I_{\lambda_{1}}\left(W_{\lambda_{1}}\right)+I_{\lambda_{2}}\left(W_{\lambda_{2}}\right)+e^{-\frac{2 \varphi\left(P_{1}, P_{2}\right)}{\varepsilon}}+o\left(e^{-\frac{2 \varphi\left(P_{1}, P_{2}\right)}{\varepsilon}}\right)\right\} .
\end{aligned}
$$

Corollary 2.7. We also have $c_{\varepsilon} \geq \varepsilon^{N}\left\{I_{\lambda_{1}}\left(W_{\lambda_{1}}\right)+I_{\lambda_{2}}\left(W_{\lambda_{2}}\right)+o(1)\right\}$.

Proof.

$$
c_{\varepsilon}=\inf _{u \in \mathcal{N}_{\varepsilon}}\left\{E_{\varepsilon, \lambda_{1}}(u)+E_{\varepsilon, \lambda_{2}}(u)\right\} \geq \inf _{u \in \mathcal{N}_{\varepsilon}^{+}} E_{\varepsilon, \lambda_{1}}(u)+\inf _{u \in \mathcal{N}_{\varepsilon}^{-}} E_{\varepsilon, \lambda_{2}}(u)
$$

this implies the result.

Lemma 2.8. As $\varepsilon \rightarrow 0$,

$$
\frac{d\left(P_{\varepsilon}^{1}, \partial \Omega\right)}{\varepsilon} \rightarrow+\infty, \frac{d\left(P_{\varepsilon}^{2}, \partial \Omega\right)}{\varepsilon} \rightarrow+\infty, \frac{\left|P_{\varepsilon}^{1}-P_{\varepsilon}^{2}\right|}{\varepsilon} \rightarrow+\infty .
$$


Proof. As $\varepsilon^{2} \Delta u_{\varepsilon}\left(P_{\varepsilon}^{1}\right) \leq 0$ it implies that $f\left(u_{\varepsilon}\left(P_{\varepsilon}^{1}\right)\right) \geq \lambda_{1} u_{\varepsilon}\left(P_{\varepsilon}^{1}\right)$ which implies that $C u_{\varepsilon}^{p-1}\left(P_{\varepsilon}^{1}\right) \geq \lambda_{1}$, hence there exists a positive constant $\beta$ such that $u_{\varepsilon}\left(P_{\varepsilon}^{1}\right) \geq$ $\beta$ and similarly we obtain that $u_{\varepsilon}\left(P_{\varepsilon}^{2}\right) \leq-\beta$. Also by Lemma 2.6,

$$
\varepsilon^{2} \int_{\Omega}\left|\nabla u_{\varepsilon}\right|^{2}+\lambda_{1} \int_{\Omega}\left(u_{\varepsilon}^{+}\right)^{2}+\lambda_{2} \int_{\Omega}\left(u_{\varepsilon}^{-}\right)^{2} \leq C \varepsilon^{N}
$$

and hence by Moser iteration we obtain $\left\|u_{\varepsilon}\right\|_{L^{\infty}(\Omega)} \leq C$.

Suppose that $\lim _{\varepsilon \rightarrow 0} \frac{d\left(P_{\varepsilon}^{1}, \partial \Omega\right)}{\varepsilon} \leq C$. By scaling $v_{\varepsilon}(x)=u_{\varepsilon}\left(\varepsilon x+P_{\varepsilon}^{1}\right)$, then $(1.2)$ reduces to,

$$
\begin{cases}\Delta v_{\varepsilon}-\lambda_{1} v_{\varepsilon}+\lambda_{2} v_{\varepsilon}^{-}+f\left(v_{\varepsilon}\right)=0 & \text { in } \Omega_{\varepsilon} \\ v_{\varepsilon}^{ \pm} \neq 0 & \text { in } \Omega_{\varepsilon} \\ v_{\varepsilon}=0 & \text { on } \partial \Omega_{\varepsilon}\end{cases}
$$

where $\Omega_{\varepsilon}=\frac{x-P_{\varepsilon}^{1}}{\varepsilon}$. Note that from (2.6), $\left\|v_{\varepsilon}\right\|_{H_{0}^{1}\left(\Omega_{\varepsilon}\right)} \leq C$; there exists $W \in$ $H^{1}\left(\mathbb{R}^{N}\right)$ we have $v_{\varepsilon} \rightarrow W$ in $H^{1}\left(\mathbb{R}^{N}\right)$ and by the Sobolev embedding theorem we have $v_{\varepsilon} \rightarrow W$ in $L_{\text {loc }}^{p}\left(\mathbb{R}^{N}\right)$. Hence $v_{\varepsilon} \rightarrow W$ point-wise almost everywhere in $\mathbb{R}^{N}$. Also by Schauder estimates, it follows that there exists $C>0$ such that $\left\|v_{\varepsilon}\right\|_{\mathcal{C}_{\text {loc }}^{2, \beta}\left(\mathbb{R}^{N}\right)} \leq C$ for some $0<\beta \leq 1$. Hence by the Ascoli-Arzela's theorem there exists $W \neq 0$ such that

$$
\left\|v_{\varepsilon}-W\right\|_{\mathcal{C}_{\mathrm{loc}}^{2}\left(\mathbb{R}^{N}\right)} \rightarrow 0 \text { as } \varepsilon \rightarrow 0
$$

where $W$ is a nontrivial solution satisfying

$$
\begin{cases}\Delta W-\lambda_{1} W+f(W)=0 & \text { in } \mathbb{R}_{+}^{N} \\ \sup W \geq \beta, W \in H^{1} & \\ W=0 & \text { on } \partial \mathbb{R}_{+}^{N}\end{cases}
$$

where $\mathbb{R}_{+}^{N}=\left\{y: y_{n}>-a\right\}$. Then by a result in [12] we obtain $W \equiv 0$, a contradiction. Similarly $\lim _{\varepsilon \rightarrow 0} \frac{d\left(P_{\varepsilon}^{2}, \partial \Omega\right)}{\varepsilon}=+\infty$. Now we prove that $\lim _{\varepsilon \rightarrow 0} \frac{\left|P_{\varepsilon}^{1}-P_{\varepsilon}^{2}\right|}{\varepsilon}=+\infty$. By applying the Schauder estimates we obtain a $C>0$ such that $\left\|\varepsilon D u_{\varepsilon}\right\|_{L^{\infty}} \leq C$. If possible let $\lim _{\varepsilon \rightarrow 0} \frac{\left|P_{\varepsilon}^{1}-P_{\varepsilon}^{2}\right|}{\varepsilon}=\delta<+\infty$. Then it easily follows that $u_{\varepsilon}\left(P_{\varepsilon}^{1}\right) \geq \beta$ and $u_{\varepsilon}\left(P_{\varepsilon}^{2}\right) \leq-\beta$ which implies that $u_{\varepsilon}\left(P_{\varepsilon}^{1}\right)-u_{\varepsilon}\left(P_{\varepsilon}^{2}\right) \geq 2 \beta$. Then

$$
2 \beta \leq\left|u_{\varepsilon}\left(P_{\varepsilon}^{1}\right)-u_{\varepsilon}\left(P_{\varepsilon}^{2}\right)\right| \leq \varepsilon\left\|D u_{\varepsilon}\right\|_{\infty} \frac{\left|P_{\varepsilon}^{1}-P_{\varepsilon}^{2}\right|}{\varepsilon} .
$$


Suppose $P_{\varepsilon}=\frac{P_{\varepsilon}^{1}-P_{\varepsilon}^{2}}{\varepsilon}$. Then along a subsequence $\left|P_{\varepsilon}\right| \rightarrow \delta \in(0,+\infty)$. Define $v_{\varepsilon}=u_{\varepsilon}\left(\varepsilon y+P_{\varepsilon}^{1}\right)$. Then $v_{\varepsilon} \rightarrow W$ in $\mathcal{C}_{\text {loc }}^{2}\left(\mathbb{R}^{N}\right)$ and $W$ satisfies

$$
\left\{\begin{array}{l}
-\Delta W+\lambda_{1} W^{+}-\lambda_{2} W^{-}=f(W) \quad \text { in } \mathbb{R}^{N} \\
W(0) \geq \beta, \quad W(P) \leq-\beta \\
W \in H^{1}\left(\mathbb{R}^{N}\right)
\end{array}\right.
$$

where $P=\lim _{\varepsilon \rightarrow 0} \frac{P_{\varepsilon}^{1}-P_{\varepsilon}^{2}}{\varepsilon}$ which implies that $W$ is a nodal solution of (2.20) and hence a critical point of the functional

$$
I_{\infty}(u)=\int_{\mathbb{R}^{N}}\left(\frac{1}{2}|\nabla u|^{2}+\frac{\lambda_{1}}{2}\left(u^{+}\right)^{2}+\frac{\lambda_{2}}{2}\left(u^{-}\right)^{2}-F(u)\right) d x
$$

and in particular we have $\left\langle I_{\infty}^{\prime}(W), W^{ \pm}\right\rangle=0$ and $W \in \mathcal{N}_{\infty}$ where

$$
\begin{aligned}
\mathcal{N}_{\infty}=\left\{u \in H^{1}\left(\mathbb{R}^{N}\right): u^{ \pm} \not \equiv\right. & 0, \int_{\mathbb{R}^{N}}\left|\nabla u^{+}\right|^{2}+\lambda_{1} \int_{\mathbb{R}^{N}}\left(u^{+}\right)^{2}=\int_{\mathbb{R}^{N}} f\left(u^{+}\right) u^{+} ; \\
& \left.\int_{\mathbb{R}^{N}}\left|\nabla u^{-}\right|^{2}+\lambda_{2} \int_{\mathbb{R}^{N}}\left(u^{-}\right)^{2}=\int_{\mathbb{R}^{N}} f\left(u^{-}\right) u^{-}\right\} .
\end{aligned}
$$

But by (2.1) we know that $\varepsilon^{N}\left(I_{\lambda_{1}}\left(W_{\lambda_{1}}\right)+I_{\lambda_{2}}\left(W_{\lambda_{2}}\right)+o(1)\right) \geq \varepsilon^{N}\left(I_{\infty}\left(W^{+}\right)+\right.$ $\left.I_{\infty}\left(W^{-}\right)+o(1)\right)$. This implies

$$
I_{\infty}\left(W^{+}\right)+I_{\infty}\left(W^{-}\right) \leq I_{\lambda_{1}}\left(W_{\lambda_{1}}\right)+I_{\lambda_{2}}\left(W_{\lambda_{2}}\right)=c_{\lambda_{1}}+c_{\lambda_{2}}
$$

where $c_{\lambda_{i}}$ is a mountain pass critical value with respect to the functional $I_{\lambda_{i}}$, i.e.

$$
c_{\lambda_{i}}=\inf _{u \in H^{1}\left(\mathbb{R}^{N}\right), u \neq 0, \int_{\mathbb{R}^{N}}|\nabla u|^{2}+\lambda_{i} \int_{\mathbb{R}^{N}} u^{2}=\int_{\mathbb{R}^{N}} f(u) u} I_{\lambda_{i}}(u) .
$$

Also it easily follows that $I_{\infty}\left(W^{+}\right)=I_{\lambda_{1}}\left(W^{+}\right) \geq c_{\lambda_{1}}, I_{\infty}\left(W^{-}\right)=I_{\lambda_{2}}\left(W^{-}\right) \geq$ $c_{\lambda_{2}}$. Since any minimizer $c_{\lambda_{i}}$ is a weak solution, we have $c_{\lambda_{1}}=I_{\lambda_{1}}\left(W^{+}\right), c_{\lambda_{2}}=$ $I_{\lambda_{2}}\left(W^{-}\right)$. Thus $W^{+}=W_{\lambda_{1}}(x-R)$ and $W^{-}=W_{\lambda_{2}}(x-S)$ for some $R, S$ in $\mathbb{R}^{N}$. The first equality implies $W^{+}>0$ on $\mathbb{R}^{N}$ which contradicts that $W$ changes sign.

Lemma 2.9. For sufficiently small $\varepsilon>0, u_{\varepsilon}$ has exactly one positive local maximum and one negative local minimum.

Proof. Note that from Lemma 2.6, we obtain that $c_{\varepsilon} \leq \varepsilon^{N}\left(I_{\lambda_{1}}\left(W_{\lambda_{1}}\right)+I_{\lambda_{2}}\left(W_{\lambda_{2}}\right)+\right.$ $o(1)$ ). Suppose it has two positive local maxima as $P_{\varepsilon}$ and $Q_{\varepsilon}$ and a negative local minimum $R_{\varepsilon}$. Then it follows similarly as in the proof of Lemma 2.8 one can show 
that $\frac{\left|P_{\varepsilon}-Q_{\varepsilon}\right|}{\varepsilon} \rightarrow+\infty, \frac{\left|Q_{\varepsilon}-R_{\varepsilon}\right|}{\varepsilon} \rightarrow+\infty$ and $\frac{\left|P_{\varepsilon}-R_{\varepsilon}\right|}{\varepsilon} \rightarrow+\infty$ as $\varepsilon \rightarrow 0$. Also note that $\frac{1}{2} f\left(u_{\varepsilon}\right) u_{\varepsilon}-F\left(u_{\varepsilon}\right) \geq 0$ by assumption (f4), and thus

$$
\begin{aligned}
c_{\varepsilon}= & E_{\varepsilon}\left(u_{\varepsilon}\right)=\int_{\Omega}\left(\frac{1}{2} f\left(u_{\varepsilon}\right) u_{\varepsilon}-F\left(u_{\varepsilon}\right)\right) d x \\
\geq & \int_{B_{\varepsilon R}\left(P_{\varepsilon}\right)}\left(\frac{1}{2} f\left(u_{\varepsilon}\right) u_{\varepsilon}-F\left(u_{\varepsilon}\right)\right)+\int_{B_{\varepsilon R}\left(Q_{\varepsilon}\right)}\left(\frac{1}{2} f\left(u_{\varepsilon}\right) u_{\varepsilon}-F\left(u_{\varepsilon}\right)\right) \\
& \quad+\int_{B_{\varepsilon R}\left(R_{\varepsilon}\right)}\left(\frac{1}{2} f\left(u_{\varepsilon}\right) u_{\varepsilon}-F\left(u_{\varepsilon}\right)\right) \\
\geq & \varepsilon^{N}\left(2 I_{\lambda_{1}}\left(W_{\lambda_{1}}\right)+I_{\lambda_{2}}\left(W_{\lambda_{2}}\right)+o(1)\right)
\end{aligned}
$$

a contradiction to Lemma 2.6. Hence $u_{\varepsilon}$ has exactly one positive maximum and one negative minimum.

Now let us define

$$
d_{\varepsilon}=\min \left\{\sqrt{\lambda}_{1} d\left(P_{\varepsilon}^{1}, \partial \Omega\right),{\sqrt{\lambda_{2}}}_{2} d\left(P_{\varepsilon}^{2}, \partial \Omega\right), \frac{\sqrt{\lambda_{1}} \sqrt{\lambda_{2}}}{\sqrt{\lambda_{1}}+\sqrt{\lambda_{2}}}\left|P_{\varepsilon}^{1}-P_{\varepsilon}^{2}\right|\right\} .
$$

Then by the above lemma $\frac{d_{\varepsilon}}{\varepsilon} \rightarrow+\infty$ as $\varepsilon \rightarrow 0$. Now let us re-scale the problem by $\bar{\varepsilon}=\frac{\varepsilon}{d_{\varepsilon}}$ and $\bar{x}=d_{\varepsilon} \bar{x}$. Then we have

$$
\Delta u-\lambda_{1} u^{+}+\lambda_{2} u^{-}+f(u)=0 \text { in } \bar{\Omega}_{d_{\varepsilon}}=\frac{\Omega}{d_{\varepsilon}} .
$$

Lemma 2.10. For any $0<\delta^{\prime}<1$, there exists a constant $C>0$ independent of $\delta^{\prime}$ such that

$$
u_{\varepsilon}^{+} \leq C e^{-\frac{\sqrt{\lambda_{1}}\left(1-\delta^{\prime}\right)\left|x-P_{\varepsilon}^{1}\right|}{\varepsilon}} \text { and } u_{\varepsilon}^{-} \leq C e^{-\frac{\sqrt{\lambda_{2}}\left(1-\delta^{\prime}\right)\left|x-P_{\varepsilon}^{2}\right|}{\varepsilon}} \forall x \in \Omega .
$$

Proof. Let $v_{\varepsilon}^{i}(y)=u_{\varepsilon}\left(\varepsilon y+P_{\varepsilon}^{i}\right)$. Then $v_{\varepsilon}^{1} \rightarrow W_{\lambda_{1}}$ in $\mathcal{C}_{\text {loc }}^{2}\left(\mathbb{R}^{N}\right)$. Also we have $W_{\lambda_{1}}(r) \leq C e^{-\sqrt{\lambda_{1}} r}$ for all $r$. Let $R=\ln \frac{C}{\zeta}$ such that $\zeta=C e^{-R}$. Then there exist an $\varepsilon_{0}>0$ such that $v_{\varepsilon}^{+}(y) \leq W_{\lambda_{1}}(y)+\zeta \leq 2 \zeta$. Let us consider the domain $\Omega^{1}=$ $\Omega \backslash B_{\varepsilon R}\left(P_{\varepsilon}^{1}\right)$ where $R>0$ is large. Hence we can choose a $\zeta>0$, independent of $\varepsilon$ such that $v_{\varepsilon}^{+} \leq C$ on $\partial B_{R}(0)$. This implies that $u_{\varepsilon}^{+} \leq 2 \zeta$ on $\partial B_{\varepsilon R}\left(P_{\varepsilon}^{1}\right)$. For any $0<\delta^{\prime}<1$, choose $\zeta$ in such a way that

$$
\frac{f\left(u_{\varepsilon}\right)}{\lambda_{1} u_{\varepsilon}^{+}}<\delta^{\prime}
$$

consider the equation with $u_{\varepsilon}>0$

$$
-\varepsilon^{2} \Delta u_{\varepsilon}+\lambda_{1} u_{\varepsilon}=\frac{f\left(u_{\varepsilon}\right)}{u_{\varepsilon}} u_{\varepsilon} \text { in } \Omega^{1} .
$$


Then we obtain,

$$
\begin{cases}-\varepsilon^{2} \Delta u_{\varepsilon}+\left(1-\delta^{\prime}\right) \lambda_{1} u_{\varepsilon} \leq 0 & \text { in } \Omega^{1} \\ u_{\varepsilon}>0 & \text { in } \Omega^{1} \\ u_{\varepsilon} \leq 2 \zeta & \text { in } \partial B_{\varepsilon}\left(P_{\varepsilon}^{1}\right) \\ u_{\varepsilon}=0 & \text { on } \partial \Omega .\end{cases}
$$

Using a comparison argument we obtain $u_{\varepsilon}^{+} \leq C e^{-\frac{\sqrt{\lambda_{1}}\left(1-\delta^{\prime}\right)\left|x-P_{\varepsilon}^{1}\right|}{\varepsilon}}$. We obtain the other estimate similarly.

\section{Lower bound of the energy expansion}

In order to obtain the greatest lower bound of the energy $E_{\varepsilon}$ we consider three cases.

Case 1. Suppose that

$$
\frac{d_{\varepsilon}}{\sqrt{\lambda_{1}} d\left(P_{\varepsilon}^{1}, \partial \Omega\right)} \rightarrow 1 \text { as } \varepsilon \rightarrow 0
$$

Note that

$$
c_{\varepsilon} \geq \inf _{u \in \mathcal{N}_{\varepsilon}^{+}} E_{\varepsilon, \lambda_{1}}(u)+\inf _{u \in \mathcal{N}_{\varepsilon}^{-}} E_{\varepsilon, \lambda_{2}}(u)
$$

We use del Pino-Felmer's symmetrization technique in [11] to conclude that

$$
E_{\varepsilon, \lambda_{1}}\left(u_{\varepsilon}^{+}\right) \geq \varepsilon^{N}\left\{I_{\lambda_{1}}\left(W_{\lambda_{1}}\right)+\frac{1}{2} e^{-2 \frac{\sqrt{\lambda_{1}}\left(d\left(P_{\varepsilon}^{1}, \partial \Omega\right)+o(1)\right)}{\varepsilon}}\right\} .
$$

We also deduce that

$$
E_{\varepsilon, \lambda_{2}}\left(u_{\varepsilon}^{-}\right) \geq \varepsilon^{N}\left\{I_{\lambda_{2}}\left(W_{\lambda_{2}}\right)+\frac{1}{2} e^{-2 \frac{\left(d_{\varepsilon}+o(1)\right)}{\varepsilon}}\right\}
$$

and as $d_{\varepsilon}=\sqrt{\lambda_{1}} d\left(P_{\varepsilon}^{1}, \partial \Omega\right)+o(1)$, we have

$$
c_{\varepsilon} \geq \varepsilon^{N}\left(I_{\lambda_{1}}\left(W_{\lambda_{1}}\right)+I_{\lambda_{2}}\left(W_{\lambda_{2}}\right)+e^{-\frac{2\left(d_{\varepsilon}+o(1)\right)}{\varepsilon}}\right) .
$$

Case 2. Suppose that

$$
\frac{d_{\varepsilon}}{\sqrt{\lambda_{2}} d\left(P_{\varepsilon}^{2}, \partial \Omega\right)} \rightarrow 1 \text { as } \varepsilon \rightarrow 0
$$

Then we argue as in Case 1. 


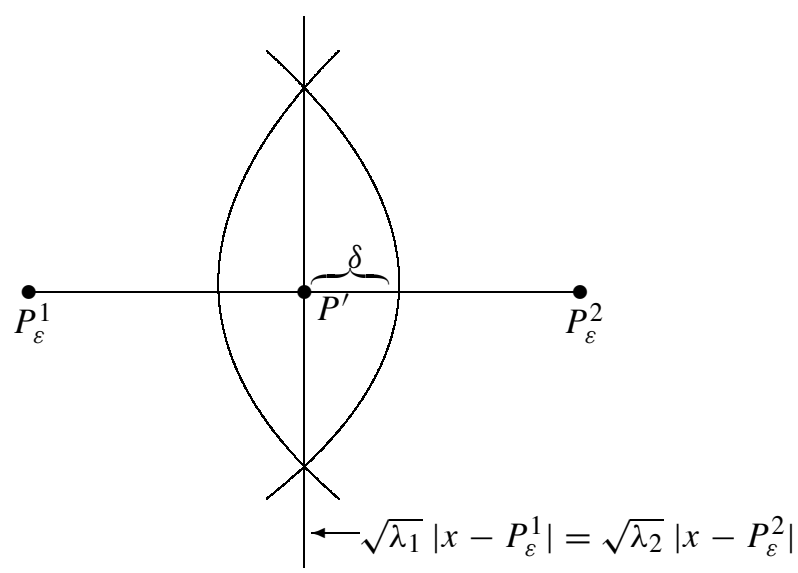

Figure 3.1. The region of intersection.

\section{Case 3.}

Suppose that

$$
d_{\varepsilon}=\frac{\sqrt{\lambda_{1}} \sqrt{\lambda_{2}}}{\sqrt{\lambda_{1}}+\sqrt{\lambda_{2}}}\left|P_{\varepsilon}^{1}-P_{\varepsilon}^{2}\right| .
$$

Then we can choose $\delta>0$ such that $d_{\varepsilon} \geq(1+5 \delta) \sqrt{\lambda_{1}} d\left(P_{\varepsilon}^{1}, \partial \Omega\right), d_{\varepsilon} \geq(1+$ $5 \delta) \sqrt{\lambda_{2}} d\left(P_{\varepsilon}^{2}, \partial \Omega\right)$. Furthermore, we define $\left|P^{\prime}-P_{\varepsilon}^{1}\right|=\frac{\sqrt{\lambda_{2}}}{\sqrt{\lambda_{1}}+\sqrt{\lambda_{2}}}\left|P_{\varepsilon}^{1}-P_{\varepsilon}^{2}\right|=d_{\varepsilon, 1}$. Then we have

$$
\left|P^{\prime}-P_{\varepsilon}^{2}\right|=\frac{\sqrt{\lambda_{1}}}{\sqrt{\lambda_{1}}+\sqrt{\lambda_{2}}}\left|P_{\varepsilon}^{1}-P_{\varepsilon}^{2}\right|=d_{\varepsilon, 2} .
$$

We consider balls $B_{d_{\varepsilon, 1}+\delta}\left(P_{\varepsilon}^{1}\right)$ and $B_{d_{\varepsilon, 2}+\delta_{2}}\left(P_{\varepsilon}^{2}\right)$, where $0<\delta \ll d_{\varepsilon, 1}$ is small and $\delta_{2} \sim \frac{\sqrt{\lambda_{2}}}{\sqrt{\lambda_{1}}} \delta$ is defined by

$$
\left(d_{\varepsilon, 1}+\delta\right)^{2}-d_{\varepsilon, 1}^{2}=\left(d_{\varepsilon, 2}+\delta_{2}\right)^{2}-d_{\varepsilon, 2}^{2}
$$

Define the intersection $\Gamma_{\varepsilon}=B_{d_{\varepsilon, 1}+\delta}\left(P_{\varepsilon}^{1}\right) \cap B_{d_{\varepsilon, 2}+\delta}\left(P_{\varepsilon}^{2}\right)$. Then the total volume of $\Gamma_{\varepsilon} \approx \delta O\left(\delta^{\frac{N-1}{2}}\right)$. Since $\Gamma_{\varepsilon}=\left(\Gamma_{\varepsilon} \cap\left\{u_{\varepsilon} \geq 0\right\}\right) \cup\left(\Gamma_{\varepsilon} \cap\left\{u_{\varepsilon} \leq 0\right\}\right)$, we either have $\left|\Gamma_{\varepsilon} \cap\left\{u_{\varepsilon} \geq 0\right\}\right| \leq \frac{1}{2}\left|\Gamma_{\varepsilon}\right|$ or $\left|\Gamma_{\varepsilon} \cap\left\{u_{\varepsilon} \leq 0\right\}\right| \leq \frac{1}{2}\left|\Gamma_{\varepsilon}\right|$.

Without loss of generality, let

$$
\left|\Gamma_{\varepsilon} \cap\left\{u_{\varepsilon} \geq 0\right\}\right| \leq \frac{1}{2}\left|\Gamma_{\varepsilon}\right|
$$

Thus

$$
\left|B_{d_{\varepsilon, 1}+\delta}\left(P_{\varepsilon}^{1}\right) \cap\left\{u_{\varepsilon}>0\right\}\right| \leq\left|B_{d_{\varepsilon, 1}+\delta}\left(P_{\varepsilon}^{1}\right)\right|-\frac{1}{2}\left|\Gamma_{\varepsilon}\right|=\left|B_{r_{\varepsilon}}(0)\right|
$$


where $r_{\varepsilon}=\left(d_{1, \varepsilon}+\delta\right)(1-\eta)$ for some $0<\eta<1$, where $\eta \sim \delta^{\frac{N+1}{2}}$. We define a smooth function

$$
\chi(x)= \begin{cases}1 & \text { if }\left|x-P_{\varepsilon}^{1}\right| \leq\left(d_{\varepsilon, 1}+\delta\right)(1-\eta) \\ 0 & \text { if }\left|x-P_{\varepsilon}^{1}\right| \geq\left(d_{\varepsilon, 1}+\delta\right)\end{cases}
$$

and $0 \leq \chi \leq 1$ and $|\nabla \chi| \leq \frac{C}{\left(d_{\varepsilon, 1}+\delta\right) \eta}$. Then the support of $u_{\varepsilon}^{+} \chi^{2}$ is contained in $B_{d_{\varepsilon, 1}+\delta}\left(P_{\varepsilon}^{1}\right)$. Multiplying (1.2) by $u_{\varepsilon}^{+} \chi^{2}$ we obtain

$$
\int_{\Omega} \varepsilon^{2} \nabla u_{\varepsilon} \nabla\left(u_{\varepsilon}^{+} \chi^{2}\right)+\lambda_{1}\left(u_{\varepsilon}^{+}\right)^{2} \chi^{2}=\int_{\Omega} f\left(u_{\varepsilon}\right) u_{\varepsilon}^{+} \chi^{2} .
$$

Now let us compute

$$
\begin{aligned}
\int_{\Omega} \varepsilon^{2} \nabla u_{\varepsilon} \nabla\left(u_{\varepsilon}^{+} \chi^{2}\right) & =\int_{\Omega} \varepsilon^{2} \nabla u_{\varepsilon}^{+} \nabla\left(u_{\varepsilon}^{+} \chi^{2}\right) \\
& =\int_{\Omega} \varepsilon^{2} \nabla u_{\varepsilon}^{+}\left\{\chi \nabla\left(u_{\varepsilon}^{+} \chi\right)+u_{\varepsilon}^{+} \chi \nabla \chi\right\} \\
& =\int_{\Omega} \varepsilon^{2}\left\{\left(\nabla\left(u_{\varepsilon}^{+} \chi\right)-u_{\varepsilon}^{+} \nabla \chi\right) \nabla\left(u_{\varepsilon}^{+} \chi\right)+u_{\varepsilon}^{+} \chi \nabla \chi \nabla u_{\varepsilon}^{+}\right\} \\
& =\int_{\Omega} \varepsilon^{2}\left\{\left|\nabla\left(u_{\varepsilon}^{+} \chi\right)\right|^{2}-u_{\varepsilon}^{+} \nabla \chi \nabla\left(u_{\varepsilon}^{+} \chi\right)+u_{\varepsilon}^{+} \chi \nabla \chi \nabla u_{\varepsilon}^{+}\right\} \\
& =\int_{\Omega} \varepsilon^{2}\left\{\left|\nabla\left(u_{\varepsilon}^{+} \chi\right)\right|^{2}-u_{\varepsilon}^{+} \chi \nabla \chi \nabla u_{\varepsilon}^{+}-\left(u_{\varepsilon}^{+}\right)^{2}|\nabla \chi|^{2}\right. \\
& \left.+u_{\varepsilon}^{+} \chi \nabla \chi \nabla u_{\varepsilon}^{+}\right\} \\
& =\varepsilon^{2} \int_{\Omega}\left|\nabla\left(u_{\varepsilon}^{+} \chi\right)\right|^{2}-\varepsilon^{2} \int_{\Omega}\left(u_{\varepsilon}^{+}\right)^{2}|\nabla \chi|^{2}
\end{aligned}
$$

where

$$
\varepsilon^{2} \int_{\Omega}\left(u_{\varepsilon}^{+}\right)^{2}|\nabla \chi|^{2} \leq C \varepsilon^{N} e^{-\sqrt{\lambda_{1}} \frac{2\left(1-\frac{\eta}{2}\right)\left(d_{\varepsilon, 1}+\delta\right)}{\varepsilon}} .
$$

On the other hand

$$
\begin{aligned}
\int_{\Omega} f\left(u_{\varepsilon}\right) u_{\varepsilon}^{+} \chi^{2} & =\int_{\Omega} f\left(u_{\varepsilon}^{+} \chi\right) u_{\varepsilon}^{+} \chi+\int_{\Omega}\left\{f\left(u_{\varepsilon}^{+} \chi\right)-f\left(u_{\varepsilon}\right) \chi\right\} u_{\varepsilon}^{+} \chi \\
& =\int_{\Omega} f\left(u_{\varepsilon}^{+} \chi\right) u_{\varepsilon}^{+} \chi+O\left(\varepsilon^{N} e^{-\frac{(p+1) \sqrt{\lambda_{1}}\left(d_{\varepsilon, 1}+\delta\right)\left(1-\frac{\eta}{2}\right)}{\varepsilon}}\right) .
\end{aligned}
$$

Note that in order to derive (3.6), we use the assumption $\left(f_{2}\right)$, Lemma $2.10,(3.3)$

$$
u_{\varepsilon}^{+} \leq C e^{-\frac{\sqrt{\lambda_{1}}\left(1-\delta^{\prime}\right)\left|x-P_{\varepsilon}^{1}\right|}{\varepsilon}}, \quad \delta^{\prime}=\frac{\eta}{2(1-\eta)},
$$


and $|\nabla \chi| \neq 0$ if $\left|x-P_{\varepsilon_{1}}\right| \geq\left(d_{\varepsilon, 1}+\delta\right)(1-\eta)$. Moreover, note that $\left\{f\left(u_{\varepsilon}^{+} \chi\right)-\right.$ $\left.f\left(u_{\varepsilon}\right) \chi\right\} u_{\varepsilon}^{+} \chi=0$ if $\chi=1$. When $\left(d_{\varepsilon, 1}+\delta\right)(1-\eta) \leq\left|x-P_{\varepsilon}^{1}\right| \leq\left(d_{\varepsilon, 1}+\delta\right)$ using (f2) we obtain

$$
\left\{f\left(u_{\varepsilon}^{+} \chi\right)-f\left(u_{\varepsilon}\right) \chi\right\} u_{\varepsilon}^{+} \chi \leq C e^{-(p+1) \frac{\sqrt{\lambda_{1}}\left(1-\delta^{\prime}\right)\left|x-P_{\varepsilon}^{1}\right|}{\varepsilon}}
$$

and hence

$$
\begin{aligned}
\int_{\Omega}\left\{f\left(u_{\varepsilon}^{+} \chi\right)-f\left(u_{\varepsilon}\right) \chi\right\} u_{\varepsilon}^{+} \chi & \leq C \varepsilon^{N} e^{-\frac{\sqrt{\lambda_{1}}(p+1)\left(d_{\varepsilon, 1}+\delta\right)\left(1-\delta^{\prime}\right)}{\varepsilon}} \\
& \leq C \varepsilon^{N} e^{-\frac{\sqrt{\lambda_{1}}(p+1)\left(d_{\varepsilon, 1}+\delta\right)\left(1-\frac{\eta}{2}\right)}{\varepsilon}} .
\end{aligned}
$$

Hence combining (3.4), (3.5) and (3.7) we have

$$
\begin{aligned}
\varepsilon^{2} & \int_{\Omega}\left|\nabla\left(u_{\varepsilon}^{+} \chi\right)\right|^{2}+\lambda_{1} \int_{\Omega}\left(u_{\varepsilon}^{+} \chi\right)^{2} \\
& =\int_{\Omega} f\left(u_{\varepsilon}^{+} \chi\right) u_{\varepsilon}^{+} \chi+O\left(\varepsilon^{N} e^{-\frac{2 \sqrt{\lambda_{1}}\left(d_{\varepsilon, 1}+\delta\right)\left(1-\frac{\eta}{2}\right)}{\varepsilon}}\right) .
\end{aligned}
$$

Let $v_{\varepsilon}=t_{\varepsilon} u_{\varepsilon}^{+} \chi$ where $t_{\varepsilon}$ is such that

$$
\varepsilon^{2} \int_{\Omega}\left|\nabla v_{\varepsilon}\right|^{2}+\lambda_{1} \int_{\Omega} v_{\varepsilon}^{2}=\int_{\Omega} f\left(v_{\varepsilon}\right) v_{\varepsilon}
$$

Now we claim that

$$
t_{\varepsilon}=1+O\left(e^{-\frac{2 \sqrt{\lambda_{1}}\left(1-\frac{\eta}{2}\right)\left(d_{\varepsilon, 1}+\delta\right)}{\varepsilon}}\right) .
$$

Define $\tilde{\sigma}:[0,+\infty) \times\left[0, \beta^{\star}\right) \rightarrow \mathbb{R}$ such that

$$
\tilde{\sigma}(t, \beta)=\int_{\Omega} f\left(t u_{\varepsilon}^{+} \chi\right) u_{\varepsilon}^{+} \chi-\int_{\Omega} f\left(u_{\varepsilon}^{+} \chi\right) u_{\varepsilon}^{+} \chi-\beta \int_{\Omega} f^{\prime}\left(u_{\varepsilon}^{+} \chi\right)\left(u_{\varepsilon}^{+} \chi\right)^{2}
$$

for some $\beta^{\star}>0$. Then $\tilde{\sigma} \in C^{1}$. Note that $\tilde{\sigma}(1,0)=0$ and

$$
\tilde{\sigma}_{t}(1,0)=\int_{\Omega} f^{\prime}\left(u_{\varepsilon}^{+} \chi\right)\left(u_{\varepsilon}^{+} \chi\right)^{2} \neq 0 .
$$

Hence by implicit function theorem, there exists a $C^{1}$ function $\beta \mapsto t(\beta)$ such that $\tilde{\sigma}(t(\beta), \beta)=0$, for small $\beta$ and $t(0)=1$. Letting $t_{\varepsilon}=1+\beta$, we have from (3.8)

$$
\beta \sim \frac{\varepsilon^{2} \int_{\Omega}\left|\nabla u_{\varepsilon}^{+} \chi\right|^{2}+\lambda_{1} \int_{\Omega}\left(u_{\varepsilon}^{+} \chi\right)^{2}-\int_{\Omega} f\left(u_{\varepsilon}^{+} \chi\right) u_{\varepsilon}^{+} \chi}{\varepsilon^{2} \int_{\Omega}\left|\nabla u_{\varepsilon}^{+} \chi\right|^{2}+\lambda_{1} \int_{\Omega}\left(u_{\varepsilon}^{+} \chi\right)^{2}-\int_{\Omega} f^{\prime}\left(u_{\varepsilon}^{+}\right)\left(u_{\varepsilon}^{+} \chi\right)^{2}} .
$$


Hence

$$
\beta \sim \frac{O\left(\varepsilon^{N} e^{-\frac{2 \sqrt{\lambda_{1}}\left(d_{\varepsilon, 1}+\delta\right)\left(1-\frac{\eta}{2}\right)}{\varepsilon}}\right)}{\int_{\Omega} f\left(u_{\varepsilon}^{+} \chi\right) u_{\varepsilon}^{+} \chi-\int_{\Omega} f^{\prime}\left(u_{\varepsilon}^{+} \chi\right)\left(u_{\varepsilon}^{+} \chi\right)^{2}}
$$

which implies $\beta=O\left(e^{-\frac{2 \sqrt{\lambda_{1}}\left(1-\frac{\eta}{2}\right)\left(d_{\varepsilon, 1}+\delta\right)}{\varepsilon}}\right)$. Then we obtain,

$$
\begin{aligned}
\frac{\varepsilon^{2}}{2} \int_{B_{d_{1, \varepsilon}+\delta}\left(P_{\varepsilon}^{1}\right)}\left|\nabla v_{\varepsilon}\right|^{2}= & \frac{\varepsilon^{2}}{2} \int_{B_{d_{1, \varepsilon}+\delta}\left(P_{\varepsilon}^{1}\right)}\left|\nabla\left(u_{\varepsilon}^{+} \chi\right)\right|^{2} \\
& +\varepsilon^{2} \beta \int_{B_{d_{1, \varepsilon}+\delta}\left(P_{\varepsilon}^{1}\right)}\left|\nabla u_{\varepsilon}^{+} \chi\right|^{2}+O\left(\beta^{2} \varepsilon^{N}\right), \\
\frac{\lambda_{1}}{2} \int_{B_{d_{1, \varepsilon}+\delta}\left(P_{\varepsilon}^{1}\right)} v_{\varepsilon}^{2}= & \frac{\lambda_{1}}{2} \int_{B_{d_{1, \varepsilon}+\delta}\left(P_{\varepsilon}^{1}\right)}\left(u_{\varepsilon}^{+} \chi\right)^{2} \\
& +\lambda_{1} \beta \int_{B_{d_{1, \varepsilon}+\delta}\left(P_{\varepsilon}^{1}\right)}\left(u_{\varepsilon}^{+} \chi\right)^{2}+O\left(\beta^{2} \varepsilon^{N}\right),
\end{aligned}
$$

and

$\int_{B_{d_{1, \varepsilon}+\delta}\left(P_{\varepsilon}^{1}\right)} F\left(v_{\varepsilon}\right)=\int_{B_{d_{1, \varepsilon}+\delta}\left(P_{\varepsilon}^{1}\right)} F\left(u_{\varepsilon}^{+} \chi\right)+\beta \int_{B_{d_{1, \varepsilon}+\delta}\left(P_{\varepsilon}^{1}\right)} f\left(u_{\varepsilon}^{+} \chi\right) u_{\varepsilon}^{+} \chi+O\left(\beta^{2} \varepsilon^{N}\right)$.

Also we have

$\varepsilon^{2} \int_{B_{d_{1, \varepsilon}+\delta}\left(P_{\varepsilon}^{1}\right)}\left|\nabla u_{\varepsilon}^{+} \chi\right|^{2}+\lambda_{1} \int_{B_{d_{1, \varepsilon}+\delta}\left(P_{\varepsilon}^{1}\right)}\left(u_{\varepsilon}^{+} \chi\right)^{2}-\int_{B_{d_{1, \varepsilon}+\delta}\left(P_{\varepsilon}^{1}\right)} f\left(u_{\varepsilon}^{+} \chi\right) u_{\varepsilon}^{+} \chi=O\left(\beta \varepsilon^{N}\right)$.

Using the above facts we have,

$$
\begin{aligned}
\frac{\varepsilon^{2}}{2} \int_{B_{d_{1, \varepsilon}+\delta}\left(P_{\varepsilon}^{1}\right)}\left|\nabla v_{\varepsilon}\right|^{2}+\frac{\lambda_{1}}{2} \int_{B_{d_{1, \varepsilon}+\delta}\left(P_{\varepsilon}^{1}\right)} v_{\varepsilon}^{2}-\int_{B_{d_{1, \varepsilon}+\delta}\left(P_{\varepsilon}^{1}\right)} F\left(v_{\varepsilon}\right) \\
=\frac{\varepsilon^{2}}{2} \int_{B_{d_{1, \varepsilon}+\delta}\left(P_{\varepsilon}^{1}\right)}\left|\nabla u_{\varepsilon}^{+} \chi\right|^{2}+\frac{\lambda_{1}}{2} \int_{B_{d_{1, \varepsilon}+\delta}\left(P_{\varepsilon}^{1}\right)}\left(u_{\varepsilon}^{+} \chi\right)^{2} \\
\quad-\int_{B_{d_{1, \varepsilon}+\delta}\left(P_{\varepsilon}^{1}\right)} F\left(u_{\varepsilon}^{+} \chi\right)+O\left(\varepsilon^{N}\left|t_{\varepsilon}-1\right|^{2}\right) \\
=\int_{B_{d_{1, \varepsilon}+\delta}\left(P_{\varepsilon}^{1}\right)}\left(\frac{1}{2} f\left(u_{\varepsilon}^{+} \chi\right) u_{\varepsilon}^{+} \chi-F\left(u_{\varepsilon}^{+} \chi\right)\right)+O\left(\varepsilon^{N}\left|t_{\varepsilon}-1\right|^{2}\right) \\
=\int_{\Omega}\left(\frac{1}{2} f\left(u_{\varepsilon}^{+}\right) u_{\varepsilon}^{+}-F\left(u_{\varepsilon}^{+}\right)\right) \\
+
\end{aligned}
$$


for some $\sigma \in(0, \min (1, p-1))$. Thus we have

$$
\begin{aligned}
E_{\varepsilon, \lambda_{1}}\left(u_{\varepsilon}^{+}\right) & \geq \inf _{\mathcal{N}_{\varepsilon}^{+}} E_{\varepsilon, \lambda_{1}, B_{d_{\varepsilon}+\delta}\left(P_{\varepsilon}^{1}\right)}(v)-C \varepsilon^{N} e^{-\frac{\sqrt{\lambda_{1}}(2+\sigma)\left(d_{\varepsilon, 1}+\delta\right)}{\varepsilon}} \\
& \geq \varepsilon^{N}\left\{I_{\lambda_{1}}\left(W_{\lambda_{1}}\right)+e^{-\frac{2 \sqrt{\lambda_{1}}\left(1-\frac{\eta}{2}\right)\left(d_{\varepsilon, 1}+\delta\right)}{\varepsilon}}\right\}-C \varepsilon^{N} e^{-\frac{\sqrt{\lambda_{1}(2+\sigma)\left(d_{\varepsilon, 1}+\delta\right)}}{\varepsilon}} \\
& \geq \varepsilon^{N}\left\{I_{\lambda_{1}}\left(W_{\lambda_{1}}\right)+\frac{1}{2} e^{-\frac{2 \sqrt{\lambda_{1}}\left(1-\frac{\eta}{2}\right)\left(d_{\varepsilon, 1}+\delta\right)}{\varepsilon}}\right\} \\
& \geq \varepsilon^{N}\left\{I_{\lambda_{1}}\left(W_{\lambda_{1}}\right)+\frac{1}{2} e^{-\frac{2\left(1-\frac{\eta}{2}\right)\left(d_{\varepsilon}+\delta\right)}{\varepsilon}}\right\} .
\end{aligned}
$$

Similarly we obtain the estimate for $E_{\varepsilon, \lambda_{2}}\left(u_{\varepsilon}^{-}\right)$. This proves the result.

Proof of Theorem 1.1. This follows from Lemma 2.6 and Section 3.

\section{References}

[1] T. BARTSCH and T. WETH, A note on additional properties of sign changing solutions to superlinear elliptic equations, Topol. Methods Nonlinear Anal. 22 (2003), 1-14.

[2] T. BARTSCH, T. WETH and M. WILlem, Partial symmetry of least energy nodal solutions to some variational problems, J. Anal. Math. 96 (2005), 1-18.

[3] V. BENCI and G. CERAMI, Multiple positive solutions of some elliptic problems via the Morse theory and the domain topology, Calc. Var. Partial Differential Equations 2 (1994), $29-48$.

[4] S. Chang, C. S. Lin, T. C. Lin and W. Lin, Segregated nodal domains of two-dimensional multispecies Bose-Einstein condensates, Phys. D 196 (2004), 341-361.

[5] M. Conti, S. TERracini and G. VerZINI, Nehari's problem and competing species system, Ann. Inst. H. Poincaré Anal. Non Linéaire 19 (2002), 871-888.

[6] M. CONTI, S. TERRACINI and G. VerZINI, An optimal partition problem related to nonlinear eigenvalues, J. Funct. Anal. 198 (2003), 160-196.

[7] M. CONTI, S. TERRACINI and G. VERZINI, Asymptotic estimates for the spatial segregation of competitive systems, Adv. Math. 195 (2005), 524-560.

[8] B. Noris, H. TAVARES, S. TERRACINI and G. VerZINI, Uniform Hölder bounds for nonlinear Schrödinger systems with strong competition, Comm. Pure Appl. Math. 63 (2010), 267-302.

[9] E. N. DANCER and Y. DU, Competing species equations with diffusion, large interactions, and jumping nonlinearities, J. Differential Equations 114 (1994), 434-475.

[10] E. N. DANCER, J. C. WEI and T. WETH, A priori bounds versus multiple existence of positive solutions for a nonlinear Schrödinger system, Ann. Inst. H. Poincaré Anal. Non Linéaire 27 (2010), 953-969.

[11] M. DEL PINO and P. FELMER, Spike-layered solutions of singularly perturbed elliptic problems in a degenerate setting, Indiana Univ. Math. J. 48 (1999), 883-898.

[12] M. ESTEBAN and P. LIONS, Existence and nonexistence results for semilinear elliptic problems in unbounded domains, Proc. Roy. Soc. Edinburgh Sect. A 93 (1982/83), , 1-14.

[13] B. GidAS, W. M. Ni and L. NiRENBERG, Symmetry and related properties via the maximum principle, Comm. Math. Phys. 68 (1979), 209-243. 
[14] T.-C. LIN and J.-C. WEI, Spikes in two coupled nonlinear Schrodinger equations, Ann. Inst. H. Poincaré Anal. Nonlinéare 22 (2005), 403-439.

[15] W. M. NI and J. WEI, On the location and profile of spike-layer solutions to singularly perturbed semilinear Dirichlet problems, Comm. Pure Appl. Math. 48 (1995), 731-768.

[16] W. M. NI and I. TAKAGI, On the shape of least-energy solutions to a semilinear Neumann problem, Comm. Pure Appl. Math. 44 (1991), 819-851.

[17] W. M. NI and I. TAKAGI Locating the peaks of least-energy solutions to a semilinear Neumann problem, Duke Math. J. 70 (1993), 247-281.

[18] EZZAT S. NOUSSAIR and J. WEI On the effect of domain geometry on the existence of nodal solutions in singular perturbations problems, Indiana Univ. Math. J. 46 (1997), 1255-1271.

[19] J. C. WEI and T. WETH, Asymptotic behavior of solutions of planar elliptic systems with strong competition, Nonlinearity 21 (2008), 305-317.

[20] J. C. WEI and T. WETH, Radial solutions and phase separation in a system of two coupled Schrödinger equations, Arch. Ration. Mech. Anal. 190 (2008), 83-106.

[21] M. Willem, "Minimax Theorems", Progress in Nonlinear Differential Equations and their Applications, Vol. 24, Birkhäuser Boston, Inc., Boston, MA, 1996.

School of Mathematics and Statistics

The University of Sydney

NSW 2006, Australia

normd@maths.usyd.edu.au

S.Santra@maths.usyd.edu.au

Department of Mathematics

The Chinese University of Hong Kong

Shatin, Hong Kong

wei@math.cuhk.edu.hk 